\title{
Fermente des menschlichen Blutes
}

\author{
XV. Mitteilung1): Der Einfluß der chemiscben Zusammensetzung von Puffern auf die quantitative Bestimmung von \\ Serumenz.ymen
}

Von W. Pilz und A. T. Boo

Aus dem Pbysiologisch-chemischen und Analytischen Labor (Leiter: Dr. W. Pila) der Ärztlichen Abteilung
(Leiter: Dr. H. Hörlein) der Farbenfabriken Bayer AG, Werk Leverkusen

(Eingegangen am 21. September 1966)

Sechs elektrophorctisch reine Enzyme wurden bei fünf Puffern unterschiedlicher Zusammensetzung, die gleiche pH-Wcrte und dieselbe Ionenstärke hatten, untersucht, wobei stets im $\mathrm{pH}$-Optimum gearbeitet wurde. Die Fehlergrenzen der verwendeten Methoden liegen dabei nicht höher als sie bei sehr empfindlichen und genauen analytischen Methoden, z. B. der Elcmentaranalysc, gefunden werden. Die Höhe der Aktivitäten ist von der chemischen Zusammensetzung des Puffers abhängig. Werden die Enzyme jedoch im Vollserum bestimrnt, ist nicht nur die Absoluthöhe der Enzyme von der Art des Puffers abhängig, sondern auch die Fehletbreite der Methoden. Eine exakte Durchrechnung war wegen der individuellen Schwankungen der Enzymaktivitäten bei den cinzelnen Versuchspersonen nicht möglich.

Six electrophoretically pure proteins were studied at their optimum $\mathrm{pH}$ in five different buffers of the same ionic strength. The limits of error of the methods employed are no wider than those found with very sensitive and exact analytical methods, e. g. elementary analysis. The activities are shown to depend on the chemical composition of the buffer. If the enzymes are determined in whole serum, however, the error of the method, as well as the activity of the enzyme, depends on the type of buffer. An exact calculation was not possible, owing to the variations in enzyme activity in individual experimental persons.

Wir konnten mehrfach feststellen, daß die Aktivität ein und desselben Enzyms von der chemischen Zusammensetzung des Puffers abhängig ist, auch wenn Ionenstärke und $\mathrm{pH}$-Wert gleich sind (z. B. (1)). Es gelingt nur in seltenen Fällen Puffersysteme zu finden, die den $\mathrm{pH}-$ Bereich von $\mathrm{pH} 4$ bis $\mathrm{pH} 10$ bestreichen ohne daß Aktivitätsänderungen von Enzymen eintreten (z. B. (2)). Außerdem fiel uns auf, daß bei der Säulenchromatographie an Ionenaustauschern das Bild des Elutionsdiagrammes stark von den verwendeten Pufferarten abhängt (3).

Wir versuchten deshalb in einer systematischen Untersuchung den Einfluß der chemischen Zusammensetzung von Puffern auf die quantitative Bestimmung von Serumenzymen festzustellen. Dabei wurde stets im $\mathrm{pH}-$ Optimum des jeweiligen Fermentes gearbeitet. Da die Aktivität auch von der Ionenstärke des Puffers abhängig sein kann (4), wurde bei allen verwendeten Puffern die Ionenstärke berechnet und ihre Herstellung so vorgenommen, daß alle Puffer gleiche Ionenstärke hatten. Als Fermente wurden im ersten Teil der Untersuchung präparativ gewonnene elektrophoretisch reine Enzyme bzw. deren wäßrige Lösungen verwendet. Im zweiten

1) Letzte Mitteilung: W. Pilz, A. T. Boo und E. Stelzi, diese Z. 5,134 (1967).
Teil wurden dieselben Enzyme bei einem Kollektiv von 250 Probanden im Volls erum bestimmt.

\section{Methodik}

Reine Serumenzyme wurden durch präparative Stärkebreielektrophorese, gegebenenfalls auch Sekundärelektrophorese, aus menschlichem Serum junger gesunder Versuchspersonen analog (1) gewonnen. Die Substrate waren in allen Fällen 1,33 ms, das Ansatzvolumen betrug $30 \mathrm{~m} /$; nur bei der Bestimmung der endogenen Lipoproteidlipase war das Substrat $0,450 \mathrm{~mm}$. Bei allen Bestimmungen wurde die Forderung nach Linearität zwischen eingesetzter Enzymmenge und gespaltenem Substrat entsprechend (5) eingehalten. Die in die Untersuchung mit verschiedenen Puffern einbezogenen Reinenzyme, ihre Substrate, pH-Optima und Bestimmungsmethoden sind in Tabelle 1 zusammengestellt. Später wurden bei 250 jungen gesunden Versuchspersonen unter denselben Bedingungen Enzymbestimmungen im Vollserum mit verschiedenen Pufferarten durchgeführt; eine Zusammenstellung der Fermente und Methoden ist in Tabelle 2 wiedergegeben.

Es wurden fünf verschiedene Puffer verwendet, die in Tabelle 3 näher beschrieben sind. Die Berechnung der Ionenstärke jedes einzelnen Puffers wurde wie folgt vorgenommen (6):

$$
\begin{gathered}
\text { Gesamte ionale Konzentration } \Gamma=\mathrm{C}_{0} \sum \mathrm{Z}_{\mathrm{i}} \mathrm{n}_{\mathrm{i}}^{2} \\
\text { Ionenstärke } \mathrm{I}=\frac{\Gamma}{2}
\end{gathered}
$$

Alle Puffer wurden so angesetzt, daß $\mathrm{I}=0,1$ war. Die mit dem TRIS-Essigsäurepuffer (18) erhaltenen Resultate wurden gleich

\begin{tabular}{|c|c|c|c|c|}
\hline Enzym & Substrat & pH-Optimum²) & Definition & Bestimmungsmethode \\
\hline Endogene Lipoproteidlipase ${ }^{1}$ ) & Tween 60 & 8,5 & HöRLEIN und PILZ (7) & $\begin{array}{l}\text { PILZ und JohaNN (8) } \\
\text { HörLEIN und PILZ (7) }\end{array}$ \\
\hline Arylesterase I & $\begin{array}{l}\beta \text {-Naphthylpropionat } \\
\text { Phenylacetat }\end{array}$ & 8,2 & $\begin{array}{l}\text { PILz (9) } \\
\text { PILz (10) }\end{array}$ & $\begin{array}{l}\text { PiLz (11) } \\
\text { Pitz und Johann (12) }\end{array}$ \\
\hline Acetylcholinesterase $\mathrm{I} \mu$ & Acetylcholin & 7,2 & PILz (1) & $P_{I L Z}(13)$ \\
\hline Acetylcholinesterase II $\mu$ & Acetylcholin & 8,6 & PILz (1) & Pilz (13) \\
\hline $\begin{array}{l}\text { Serumaliesterasen (Summe der } \\
11 \text { Enzyme) }\end{array}$ & $\begin{array}{l}\text { Benzoylcholin } \\
\alpha \text {-Naphthylpropionat }\end{array}$ & 8,6 & $\begin{array}{l}\text { PILZ (1) } \\
\text { PILZ und HöRLEIN (14) }\end{array}$ & $\begin{array}{l}\text { Pisiz (15) } \\
\text { PILz und JohanN (16) }\end{array}$ \\
\hline
\end{tabular}

Tab. 1. Untersuchte elektrophoretisch reine Enzyme aus menschlichem Serum

1) Endogene Lipoproteidlipase = CE (Clearing-Enzym 7); vgl. auch PILZ und HöRLEIN (17).

1) Endogene Lipoproteidlipase
2 ) Es wurde stets im pH-Optimum der Enzyme gearbeitet. 
Tab. 2. Im menschlichen Vollserum bestimmte Enzyme

\begin{tabular}{|c|c|c|c|}
\hline Enzym & Substrat & pH-Wert bei der Untersuchung ${ }^{2}$ ) & Bestimmungsmethode \\
\hline Endogene Lipoproteidlipase') & Tween 60 & 8,5 & $\begin{array}{l}\text { PILZ und JOHANN (8) } \\
\text { HöRLEIN und PILZ (7) }\end{array}$ \\
\hline Arylesterase I & $\beta$-Naphthylpropionat & 8,2 & $\begin{array}{l}\text { Pilz }(9,11) \\
\text { Pilz und JohanN (12) }\end{array}$ \\
\hline $\begin{array}{l}\text { Acetylcholinesterase } \\
\text { Aliesterase }\end{array}$ & $\begin{array}{l}\text { Acetylcholin } \\
\text { Benzoylcholin } \\
\text { a-Nyphthylpropionat }\end{array}$ & $\begin{array}{l}8 ; 6 \\
8,6\end{array}$ & $\begin{array}{l}\text { Pilz (13) } \\
\text { Pilz (15) } \\
\text { Pilz und JohanN (16) }\end{array}$ \\
\hline
\end{tabular}

1) Endogene Lipoproteidlipase = CE (Clearing-Enzym 7); vgl. auch PILz und HöRLEIN (17).

$\Rightarrow$ Es wurde stets im pH-Optimum der Enzyme gearbeitet.

Tab. 3. Verwendete Puffersysteme

\begin{tabular}{lll}
\hline Bezeichnung (Kennbuchstabe) & Chemische Zusammensetzung & pH-Bereich \\
\hline TRIS-Essigsäure (A) & Tris(hydroxymethyl)aminomethan, Essigsäure \\
Borat (B) & Borsäure, $\mathrm{KCl}, \mathrm{NaOH}$ & $6,7-9,1$ \\
Citronensäure-Phosphat (C) & Citronensäure, Phosphorsäure, NaOH, Borsäure \\
Veronal (D) & Veronalnatrium, HCl & $\mathbf{8 , 2 - 1 0}$ \\
Borax-Phosphat (E) & Natriumtetraborat, Kaliumbiphosphat \\
\hline
\end{tabular}

$100 \%$ gesetzt und alle anderen Ergebnisse auf diesen Wert bezogen. In allen Fällen wurde im pH-Optimum des jeweiligen Enzyms gearbeitet. Die pH-Werte aller Puffer und Ansätze wurden elektrometrisch kontrolliert.

\section{Ergebnisse}

Zunächst wurden Lösungen der elektrophoretisch reinen Enzyme unter Verwendung des Puffers TRISEssigsäure (18) (Kennbuchstabe A) $25 \mathrm{mal}$ bestimmt; die Bestimmungen wurden in wechselnder Reihenfolge von 3 Untersuchern vorgenommen. Die Resultate sind in Tabelle 4 zusammengestellt. Desgleichen wurden die Reinenzyme auch mit den Puffern B, C, D und E bestimmt, wobei die in den Abbildungen 1 und 2 wiedergegebenen Resultate erhalten wurden.

Die Bestimmung der Enzyme im Vollserum von 250 Versuchspersonen wurde in derselben Weise durchgeführt. Die Resultate sind in den Abbildungen 3-8 zusammengestellt. Die Werte stellen das arithmetische Mittel aus jeweils 250 Doppelbestimmungen dar; daraus

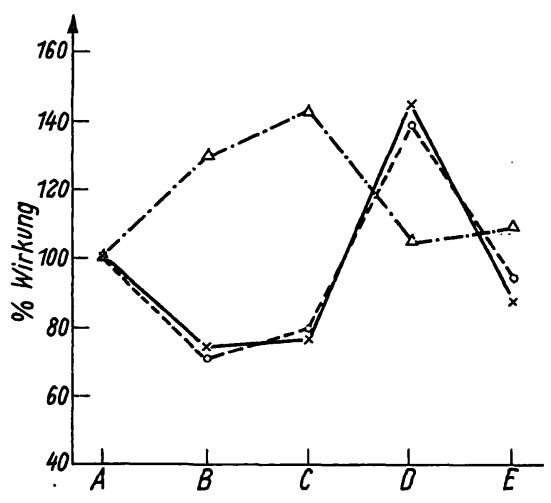

Abb. 1

Einfluß der chemischen Pufferzusammensetzung auf die Aktivität von Reinenzymen

$\times-\times$ Acetylcholinesterase I $\mu ; O--O$ Acetylcholinesterase II $\mu ; \Delta---\Delta$ Aliesterase (Substrat: Benzoylcholin); Abszisse: vgl.
Tab. 3; Ordinate: \% Wirkung $(\mathrm{A}=100 \%)$ ergibt sich die eingezeichnete je nach Enzym und Pufferart stark unterschiedliche Fehlerbreite.

\section{Diskussion}

Die Angaben über die Höhe der Enzymaktivität und die Fehlerbreite in Tabelle 4 beziehen sich nur auf Analysen, die mit TRIS-Essigsäurepuffer ausgeführt wurden. Sofern man Reinenzyme verwendet, beeinflußt die chemische Zusammensetzung der Puffer wohl die Höhe der Aktivität, nicht aber die Fehlerbreite der Methode (vgl. Abb. 1 und 2). Einzelwerte (entsprechend Tab. 4) mußten daher nicht aufgeführt werden. Die Fehlerbreite der verwendeten Methoden ist nicht größer als man sie von sehr genauen analytischen Methoden (z. B. Elementaranalyse usw.) erwartet. Dies gilt jedoch nur dann, wenn elektrophoretisch reine Enzyme verwendet werden.

Die Bestimmung der gleichen Enzyme nach denselben Methoden im Vollserum zeigt außer einer deutlichen

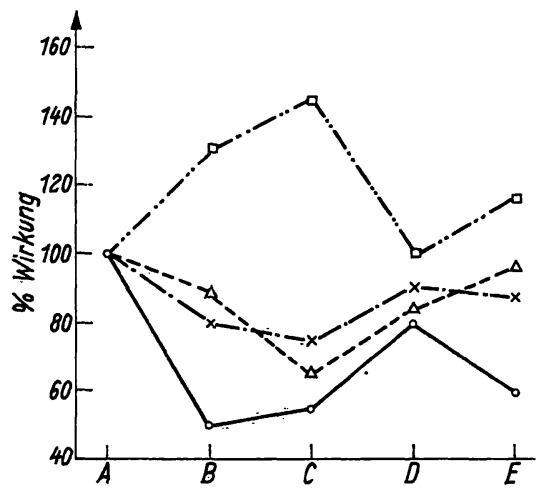

Abb. 2

Eingflụß der chemischen Pufferzusammensetzung auf die Aktivität von Reinenzymen

O_O endogene Lipoproteidlipase (CE, Substrat: Tween 60); esterase (Substrase I (Substrat: Phenylacetat; $\square-. .-.-\square$ Al Ordinate: \% Wirkung $(A=100 \%)$ 
Tab. 4. Aktivität von Lösungen der elektrophoretisch reinen Enzyme und Fehlergrenzen der Bestimmungsmethoden')

\begin{tabular}{|c|c|c|c|c|c|}
\hline Enzym & pH-Optimum & Substrat & $\begin{array}{l}\text { Methode } \\
\text { (Zitat) }\end{array}$ & $\begin{array}{l}\left.\text { Aktivität }{ }^{2}\right) \\
(\mu \mathrm{A} q / \mathrm{m} l / \mathrm{Std} .)\end{array}$ & $\underset{\%}{\text { Fehler }}$ \\
\hline Endogene Lipoproteidlipase?) & 8,5 & $\begin{array}{l}\text { Tween } 60 \\
\text { mit Serum aktiviertes Maisöl }\end{array}$ & $\begin{array}{l}(7) \\
(8)\end{array}$ & $\begin{array}{l}32,8 \\
33,2\end{array}$ & $\begin{array}{l} \pm 2,1 \\
\pm 0,3\end{array}$ \\
\hline Arylesterase I & 8,2 & $\begin{array}{l}\beta \text {-Naphthylpropionat } \\
\text { Phenylacetat }\end{array}$ & (11) & 12,6 & $\begin{array}{l} \pm 0,3 \\
+0,3\end{array}$ \\
\hline Acetylcholinesterase $\mathbf{I} \mu$ & 7,2 & Acetylcholin & (13) & 9,6 & $\pm 0,18$ \\
\hline Acetylcholinesterase $11 / 1$ & 8,6 & Acetylcholin & (13) & 9,2 & $\pm 0,19$ \\
\hline & & a-Naphthylpropionat & (16) & 14,1 & $\pm 0,11$ \\
\hline
\end{tabular}

1) Alle Analysen wurden im jeweiligen pH-Optimum mit TRIS-Essigsäure ausgeführt.

2) Arithmetisches Mittel aus 25 Analysen.

3) Endogene Lipoproteidlipase = CE (Clearing-Enzym, 7); vgl. auch $(17,2)$

) Summe der 11 unspezifischen Serumenzyme nach (1) in physiologischer Zusammensetzung.

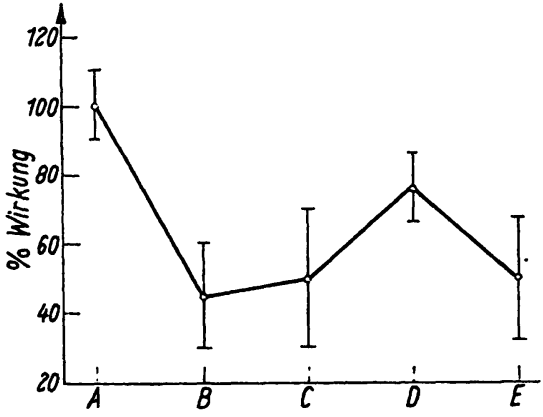

Abb. 3

Einfluß der chemischen Pufferzusammensctzung auf die Aktivität der endogenen Lipoproteidlipase. (CE; Substrat: Tween 60).

Untersuchung an 250 Probanden (VollOrdinate: \% Wirkung $(\dot{A}=100 \%)$

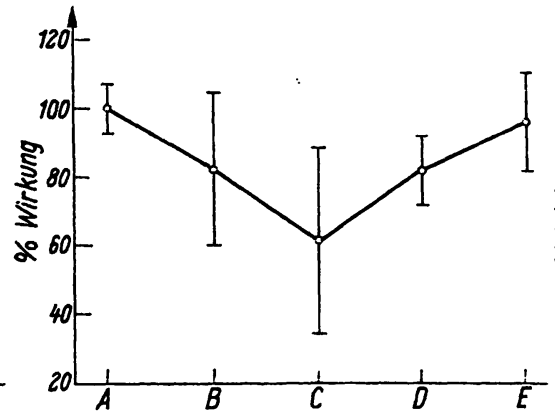

Abb. 4

Einfluß der chemischen Pufferzusammensetzung auf die Aktivität der Arylesterase I (Substrat: $\beta$-Naphthylpropionat)

Untersuchung an 250 Probanden (Vollserum). Abszisse: vgl. Tab. 3; Ordi-

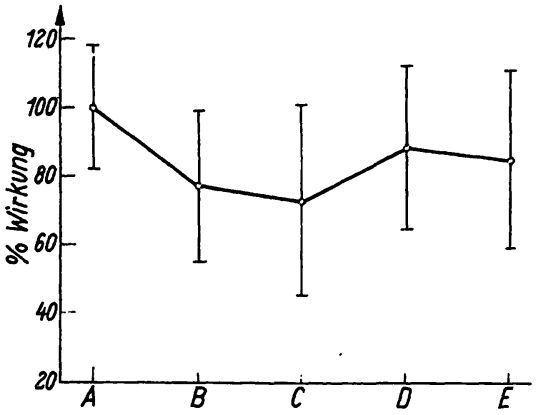

Abb. 5

Einfluß der chemischen Pufferzusammensetzung auf die Aktivität der Arylesterase I (Substrat: Phenylacetat)

Untersuchung an 250 Probanden (Vollserum). Abszisse: vgl. Tab. 3; Ord
nate: $\%$ Wirkung $(A=100 \%)$

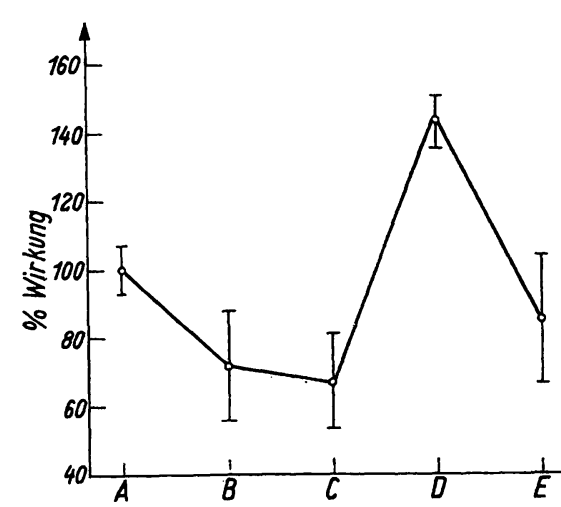

Abb. 6

Einfluß der chemischen Pufferzusammensetzung auf die Aktivität der Acetylcholinesterase (Substrat: Acetylcholin)

Untersuchung an 250 Probanden (Vollserum). Abszisse: vgl. Tab. 3 ; Ordinate: \% Wirkung ( $\mathrm{A}=100 \%$ )

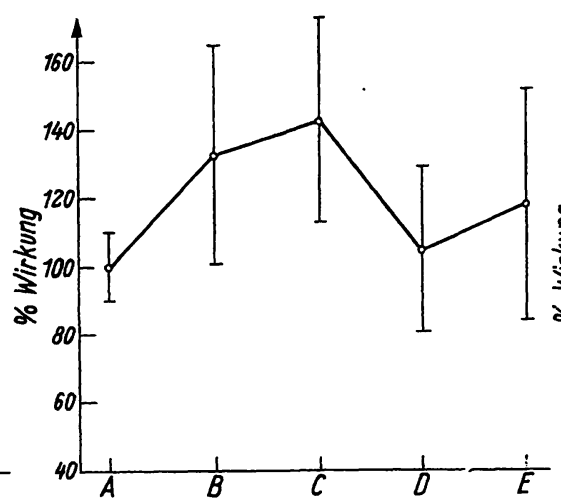

Abb. 7

Einfluß der chemischen Pufferzusammensetzung auf die Aktivität der Aliesterase (Substrat: Benzoylcholin)

Untersuchung an 250 Probanden (Vollserum). Abszisse: vgl. Tab. 3; Ordinate: \% Wirkung (A $=100 \%)$

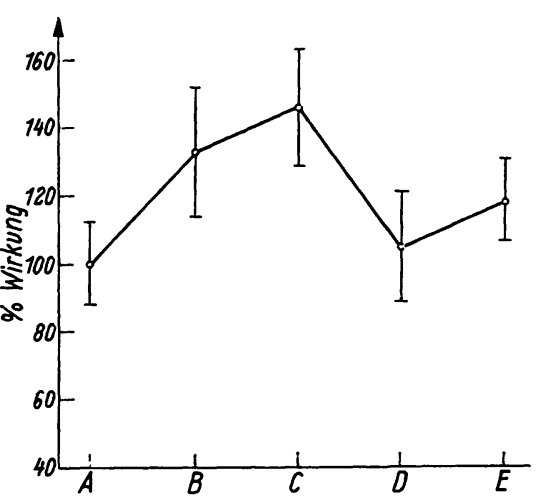

Abb. 8

Einfluß der chemischen Pufferzusammensetzung auf die Aktivität der Aliesterase (Substrat: $\alpha$-Naphthylpropionat)

Untersuchung an 250 Probanden (Vollnate: $\%$ Wirkung $(A=100 \%)$
Abhängigkeit der Aktivität von der chemischen $\mathrm{Zu}-$ sammensetzung des Puffers zusätzlich eine starke $\mathrm{Ab}$ hängigkeit der Feblerbreite von der Pufferart. So sind die Fehler bei Verwendung von TRIS-Essigsäurepuffer bei allen Fermenten am geringsten. Bei den meisten Enzymen wird auch mit Veronalpuffer eine verhältnismäßig geringe Fehlerbreite erhalten. Alle Puffer die Borsäure enthalten, verursachen bedeutend größere Fehlerbreiten. Mit den Abbildungen 3-8 ist einwandfrei bewiesen, daß sowohl Enzymaktivität als auch Fehlerbreite stark von der chemischen Zusammensetzung des verwendeten Puffers abhängig sind. Die große Fehlerbreite kann nicht 
allein durch die individuellen Unterschiede der Enzymaktivitäten geklärt werden, da z. B. im Falle der Benzoylcholinhydrolyse durch Vollserum (Abb. 7) der Fehler mit TRIS-Essigsäure $\pm 10 \%$ beträgt, während er mit anderen Puffern ein Mehrfaches davon ausmacht. Ähnlich sind die Verhältnisse z. B. bei der endogenen Lipoproteinlipase (Abb. 3). Auch hier beträgt der Fehler beim TRIS-Essigsäurepuffer $\pm 10 \%$; derselbe Wert wurde bei verminderter Absoluthöhe mit Veronalpuffer gefunden, während der Fehler mit CitronensäurePhosphatpuffer $20 \%$ beträgt.

Damit ist erwiesen, daß sowohl die Absoluthöhe der Enzymaktivität als auch die Fehlerbreite der jeweiligen
Bestimmungsmethode von der chemischen Zusammensetzung des Puffers abbängt, sofern nicht mit Reinfermenten gearbeitet wird. Es wäre daher wünschenswert, daß bei Beschreibungen von Enzymen und der Bekanntgabe von Methoden der enzymatischen Analyse, größeres Augenmerk auf die chemische Zusammensetzung der Puffer gelegt würde, als dies bisher der Fall war. Speziell für die klinische Chemie ist $z u$ fordern, daß bei allen Methoden außer $\mathrm{pH}$-Wert und Ionenstärke auch die genaue chemische Zusammensetzung des verwendeten Puffers sowie die Fehlergrenze der Methode bei den gewählten Bedingungen anzugeben ist.

\title{
Literatur
}

1. PrLz, W., Hoppe-Seyler's Z. physiol. Chem. 345, 80 (1966). 2. Pizz, W. und I. JohanN, Hoppe-Seyler's Z. physiol. Chem., im Druck. - 3. Pilz, W., H. Hörlein und E. StelzL, Hoppe-Seyler's Z. physiol. Chem. 345, 65 (1966). - 4. Przz, W., I. JohanN und E. Stelzl, Z. analyt. Chem. 215, 260 (1966). - 5. Pilz, W., diese Z.3, 89 (1965). - 6. Eucken, A. und E. WICKE, Grundriß der physikalischen Chemie, S. 547, Verlag Geest u. Portig, Leipzig (1958). - 7. Hörleis, H. und W. PILZ, Hoppe-Seyler's Z. physiol. Chem. 327, 256 (1962). - 8. Prlz, W. und I. Johann, Z. analyt. Chem. 218, 426 (1966). - 9. PrIz, W., Hoppe-Seyler's Z. physiol. Chem. 328, 1 (1962). - 10. PrLz, W., Hoppe-Seyler's Z. physiol. Chem. 328, 247 (1962). - 11. PrLz, W., Mikrochim. Acta (Wien) 1961, S. 614. - 12. Pilz, W. und I. JohanN, Z. analyt. Chem. 212, 410 (1965). - 13. Pilz, W., Methoden der Enzymatischen Analyse, S. 765, Verlag Chemie GmbH, Weinheim/Bergstr. (1962). - 14. Prlz, W. und H. Hördern, Hoppe-Seyler's Z. physiol. Chem. 339, 157 (1964). - 15. PiLz, W., Zschr. exper. Med. 132, 310 (1959). - 16. Priz, W. und İ. JohanN, Z. analyt. Chem. 210, 113 (1965). - 17. Przz, W. und H. HöRLEIN, Hoppe-Seyler's Z. physiol. Chem. 330, 212 (1963). - 18. Pilz, W. und I. Johann, Z. analyt. Chem. 215, 105 (1965).
Dr. W. Pilz

Physiol.-chem. u. analyt. Labor der ärztl. Abteilung

559 Leverkusen-Bayerwerk

\section{Fractionation and determination of 17 -ketosteroids by means of column and thin layer chromatography}

\author{
By L. E. Böttiger and B. P. LisBoA \\ From King Gustaf Vth Research Institute, (Head: G. Birke, MD), Stockholm, Siveden
}

(Eingegangen am 2. August 1966)

\begin{abstract}
A method is described for the fractionation of urinary 17-ketosteroids by use of gradient elution chromatography on aluminium oxide followed by one-dimensional multiple thin layer chromatography on silica gel G.

Androsterone, etiocholanolone, $11 \beta$-hydroxy-androsterone, 11-oxoetiocholanolone, 11 $\beta$-hydroxyetiocholanonole, 11-oxo-androsterone and a fraction containing dehydroepiandrosterone together with its rearranged product, $i$-androstanolone, were determined by means of the ZiMMERMANN reaction. The individual steroids were characterized by means of one- and two-dimensional thin layer chromatography using single and multiple runs, and by several "in situ" developed colour reactions. The method has been developed for sulphuric acid hydrolysis followed by continuous ether extraction as well as for enzymatichydrolysis; the results with the different hydrolysis procedures have been discussed. Results are presented for the fractionation of fifteen normal urines after sulphuric acid hydrolysis. The ratio between the amounts of etiocholanolone and androsterone obtained by this method has been compared with that found after additional oxidation of their $\Delta^{9}\left({ }^{11}\right)$ dehydro-derivatives.
\end{abstract}

Es wird eine Methode zur Fraktionierung der 17-Ketosteroide des Harnes durch Gradienten-Elutions-Chromatographie an Aluminiumoxyd mit anschließender mehrfacher eindimensionaler Dünnschichtchromatographie auf Silikagel G beschrieben. Mittels ZimmermanNReaktion wurden folgende Substanzen bestimmt: Androsteron, Ätiocholanolon, 11 $\beta$-Hydroxyandrosteron, 11-Oxyätiocholanolon, 11 $\beta$ Hydroxyäthiocholanolon, 11-Oxyandrosteron und eine Fraktion enthaltend Dehydroepiandrosteron zusammen mit seinem Umwandlungsprodukt i-Androstanolon. Die einzelnen Steroide wurden charakterisiert durch ein- und zweidimensionale Dünnschichtchromatographie - und zwar einfach und mehrfach - und durch einige „in situ“ entwickelte Farbreaktionen. - Die Methode wurde sowohl für schwefelsaure Hydrolyse mit kontinuierlicher Äther-Extraktion wie auch für enzymatische Hydrolyse entwickelt. Die Ergebnisse mit den verschiedenen Hydrolyse-Verfahren werden diskutiert. Es werden die Ergebnisse der Fraktionierung von 15 Normalharnen nach Schwefelsäure-Hydrolyse mitgeteilt. Das nach dieser Methode ermittelte Verhältnis Ätiocholanolon/Androsteron wird mit demjenigen verglichen, das nach zusätzlicher Oxydation ihrer $\Delta^{9}\left({ }^{12}\right)$-Dehydro-Derivate gefunden wird. 